\title{
Semiquantitative hormone receptor level influences response to trastuzumab-containing neoadjuvant chemotherapy in HER2-positive breast cancer
}

Rohit Bhargava $^{1}$, David J Dabbs ${ }^{1}$, Sushil Beriwal ${ }^{2}$, Isil A Yildiz ${ }^{1}$, Preeti Badve ${ }^{1}$, Atilla Soran ${ }^{3}$, Ronald R Johnson ${ }^{3}$, Adam M Brufsky ${ }^{4}$, Barry C Lembersky ${ }^{4}$, Kandace P McGuire ${ }^{3}$ and Gretchen M Ahrendt ${ }^{3}$

${ }^{1}$ Department of Pathology, Magee-Womens Hospital of UPMC, Pittsburgh, PA, USA; ${ }^{2}$ Department of Radiation Oncology, Magee-Womens Hospital of UPMC, Pittsburgh, PA, USA $;{ }^{3}$ Department of Surgery, Magee-Womens Hospital of UPMC, Pittsburgh, PA, USA and ${ }^{4}$ Department of Medical Oncology, Magee-Womens Hospital of UPMC, Pittsburgh, PA, USA

Pathologic complete response to neoadjuvant chemotherapy without trastuzumab in hormone receptornegative/HER2 + tumors is seen in $\mathbf{2 7 - 4 5 \%}$ of cases. In contrast, estrogen receptor (ER) +/HER2 + tumors demonstrate pathologic complete response in $\sim 8 \%$ of cases and is generally limited to weak-to-moderate ER + /HER2 + tumors. It is speculated that addition of trastuzumab to neoadjuvant chemotherapy regimen will increase the pathologic complete response rates in all HER2 + tumors. A list of HER2 + patients who received neoadjuvant chemotherapy (with trastuzumab) in the years 2007-2010 was obtained from our hospital database. The 104 HER2 + tumors were classified into three groups based on semiquantitative hormone receptor and HER2 results as follows: ERBB2 (ER-/PR-[H-score $\leq 10] / H E R 2+$ ), Luminal B-HER2 Hybrid (LBHH; weak to moderate ER + [H-score 11-199]/HER2 +), and Luminal A-HER2 Hybrid (LAHH; strong ER + [H-score $\geq 200] /$ HER2 +). Pathologic complete response was defined as absence of invasive carcinoma in the resection specimen and in the lymph nodes. Percentage tumor volume reduction was also calculated based on pretherapy size and detailed evaluation of the resection specimen. In all, $52 \%$ (25 of 48 cases) of ERBB2 tumors showed pathologic complete response, which was significantly higher than the pathologic complete response rate in LBHH (33\%; 10 of 30$)$ and LAHH ( $8 \% ; 2$ of 26) tumors. Average percentage tumor volume reduction was also highest in ERBB2 tumors (86\%), followed by LBHH $(74 \%)$ and LAHH $(64 \%)$ tumors. We conclude that addition of trastuzumab to neoadjuvant chemotherapy regimen significantly increases the pathologic complete response rates in all HER2 + tumors. However, the benefit of trastuzumab is highest in ER-negative tumors and progressively decreases with increase in tumor ER expression. This information can be utilized to counsel patients considered for neoadjuvant chemotherapy and the same principle could be applied in the adjuvant setting.

Modern Pathology (2011) 24, 367-374; doi:10.1038/modpathol.2010.209; published online 19 November 2010

Keywords: HER2 + tumors; pathologic complete response and tumor volume reduction; trastuzumab-containing neoadjuvant therapy

Preoperative or neoadjuvant chemotherapy is increasingly used in breast carcinoma since studies

Correspondence: Dr R Bhargava, MD, Department of Pathology, Magee-Womens Hospital, University of Pittsburgh Medical Center, 300 Halket Street, Pittsburgh PA 15213, USA.

E-mail: rbhargava@mail.magee.edu

Received 14 September 2010; revised 7 October 2010; accepted 13

October 2010; published online 19 November 2010 have shown no difference in survival benefits with respect to timing of chemotherapy (before or after surgery). ${ }^{1-4}$ Moreover, the benefits of neoadjuvant chemotherapy from surgical and investigational standpoint are manifold. A few years ago, neoadjuvant chemotherapy was used only in locally advanced breast cancers that were deemed inoperable. ${ }^{5}$ More recently, it has been increasingly used for tumors that are resectable, but the intent is to 
reduce the tumor size by neoadjuvant chemotherapy and subsequently remove a smaller portion of breast tissue than would otherwise be removed at primary surgery. ${ }^{6-8}$ Breast cancer is a heterogeneous disease at morphological, immunohistochemical and even at molecular level. Therefore, some breast carcinomas respond completely to neoadjuvant chemotherapy and others show minimal or no response. From a surgeon's perspective, it is important to know in advance if they will be able to convert a mastectomy to breast-conserving surgery at the time of initial evaluation.

Recent studies have shown that pathologic complete response is seen in significant proportion of estrogen receptor (ER)-negative tumors and is only rarely observed in ER-positive disease..$^{9-12}$ In our previous study of 359 cases, pathologic complete response was observed in $33 \%$ of ER-/PR-/HER2 + cases, $30 \%$ of triple-negative cases and in $<10 \%$ of ER + cases. Among the ER + tumors, pathologic complete response was predominantly seen in tumors that co-expressed HER2 with low-to-moderate ER expression. ${ }^{9}$ Although pathologic complete response was seen mainly in ER-negative tumors, tumor volume reduction was seen in most carcinomas and appeared to be inversely related to tumor ER expression (especially in HER2 + tumors). In these previous studies, including our previous study, most patients were treated with chemotherapy regimens that did not include trastuzumab. ${ }^{9,11,13}$ This is because trastuzumab has been only rarely used in neoadjuvant chemotherapy regimens before 2006, where its use was limited to clinical trials (MD Anderson trial, NOAH trial and GeparQuattro trial). ${ }^{14-16}$ Recently, trastuzumab is increasingly used preoperatively in HER2 + breast carcinoma, often as TCH regimen (Taxotere $\mathbb{R}$, carboplatinum, Herceptin ( ) ) or as AC followed by TH (ie, adriamycin, cyclophosphamide followed by taxane and herceptin) or in some other combination. ${ }^{17,18}$ Pathologic complete response to neoadjuvant chemotherapy without trastuzumab in hormone receptor-negative/HER2 + tumors is seen in $27-45 \%$ of cases. ${ }^{9,10,12}$ In contrast, ER + /HER2 + tumors demonstrate pathologic complete response in $\sim 8 \%$ of cases and is generally limited to weak-tomoderate ER + /HER2 + tumors. $^{9}$ It is speculated and also shown in recently concluded clinical trials that addition of trastuzumab to neoadjuvant chemotherapy regimen will increase the pathologic complete response rates in all HER2 + tumors. ${ }^{14-16,19}$ However, even in these clinical trials the effect of trastuzumab on different subsets of HER2 + tumors has not been addressed. The primary aim of this study was to analyze the rate of pathologic complete response in different types of HER2 + tumors. We also objectively calculated residual tumor volume and studied percentage tumor volume reduction to trastuzumab-containing chemotherapy in different categories of HER2 + tumors.

\section{Materials and methods}

A list of HER2 + patients who received neoadjuvant chemotherapy in the years 2007-2010 was obtained from our hospital database. All patients received trastuzumab-containing chemotherapy most often as TCH regimen (taxotere, carboplatinum and herceptin). The 104 HER2 + tumors were classified into three groups based on semiquantitative hormone receptor and HER2 results as follows: ERBB2 (hormone receptor-negative and HER2 + ), Luminal B-HER2 Hybrid (LBHH; weak/moderate ER + and HER2 +) and Luminal A-HER2 Hybrid (LAHH; strong ER + and HER2 + ). Examples of these three tumor types are shown in Figure 1. Hormone receptor expression was categorized as negative, weak/moderate expression and strong expression based on semiquantitative modified H-score method, where the score ranges from 0 to $300 .^{20,21}$ An $\mathrm{H}$-score of $\geq 200$ was considered as strong expression, scores of 11-199 was considered as weak/ moderate expression and an $\mathrm{H}$-score of $\leq 10$ was considered a negative result. HER2 was examined either with immunohistochemistry or fluorescence in situ hybridization (FISH). HER2 immunohistochemical scores of 0 and $1+$ (ie, no staining or weak incomplete membranous staining in any proportion of tumor cells) were considered as negative. Immunohistochemical score of $3+$ (strong membranous reactivity in $>30 \%$ of the tumor cells) was considered as positive. HER2 immunohistochemical $2+$ (weak-to-moderate circumferential membranous reactivity in at least $10 \%$ of the tumor cells and strong membranous reactivity in $\leq 30 \%$ of tumor cells) cases were considered as positive only when unequivocally amplified by FISH. An equivocal FISH result was considered as a negative result in this study. Ki-67 results were available only on 21 cases. The labeling index was determined by manually estimating the percentage of positive tumor cells. All invasive tumor cells were taken into account for estimating the Ki-67 labeling index. Immunohistochemical stains for ER (clone SP1; Ventana Medical Systems, Tucson, AZ, USA), PR (clone 1E2; Ventana), HER2 (clone 4B5; Ventana) and in recent cases Ki-67 (clone 30-9; Ventana) were performed at the time of initial diagnosis. All antibodies were received predilute and the pretreatment consisted of cell conditioning system 1 (CC1 from Ventana). The antigen-antibody complexes were detected using iVIEW/DAB detection kit on BenchMark XT (Ventana).

Pathologic complete response was defined as absence of invasive carcinoma in the resection specimen and in the lymph nodes. Percentage tumor size reduction was also calculated based on pretherapy size and detailed evaluation of the resection specimen. For the years 2007, 2008 and early part of 2009 , the evaluation of tumor volume reduction was somewhat subjective, as a standard protocol was not universally followed. Nevertheless, the examination 

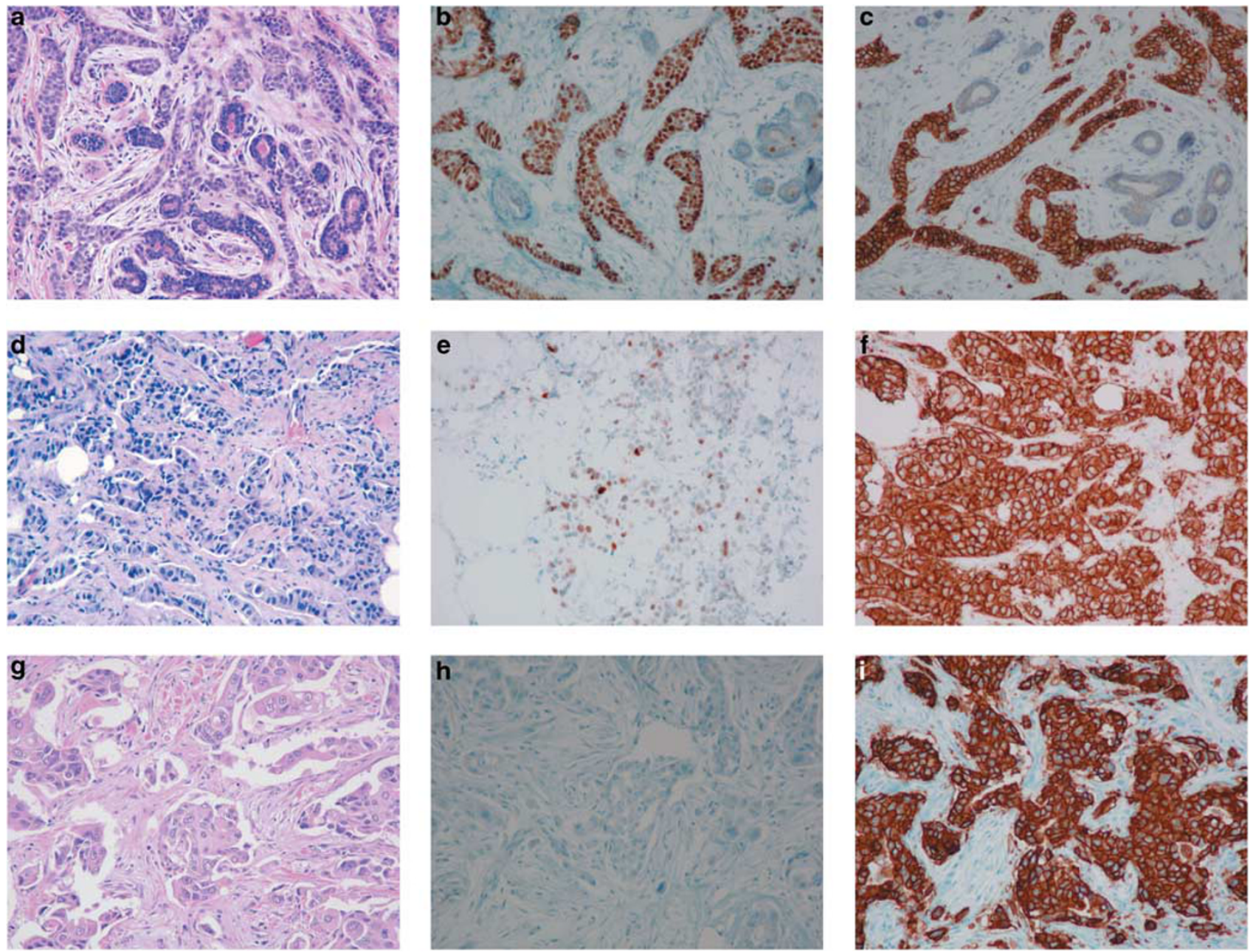

Figure 1 Example of a Luminal A-HER2 hybrid tumor (a) with strong estrogen receptor expression (b) and HER2 positivity (c). Example of a Luminal B-HER2 hybrid tumor (d) with weak/moderate estrogen receptor expression (e) and HER2 positivity (f). Example of an ERBB2 tumor (g), negative for estrogen receptor (h) and HER2 positivity (i).

was thorough and the best estimate for tumor volume reduction was deduced by detailed examination of gross and microscopic description in the pathology reports. Moreover, tissue handling and fixation has already been standardized since 2007 at our institution. For the later half of 2009 and 2010 cases, a standard method for tumor size/volume reduction was followed. Specifically, the largest dimension of the gross tumor-bed/fibrotic area identified on gross examination was noted in the gross description of the pathology report. This area was either entirely submitted (if small, ie, $\leq 3 \mathrm{~cm}$ ) or sampled extensively (if large, ie, $>3 \mathrm{~cm}$ ), with sections serially submitted at $0.5 \mathrm{~cm}$ interval along the largest dimension. The entire region was submitted regardless of the size if no tumor was detected on initial sections. The tumor cellularity of the resection specimen was compared with the pretherapy biopsy. Specifically, the pretherapy biopsy was screened for de novo sclerosis and necrosis. If these areas were present in pretherapy biopsy, then similar areas in post-therapy resection specimens were not counted toward therapy-related changes. After excluding these de novo changes, the resection specimen showing treatment-related fibrosis was compared with the cellularity of pretherapy biopsy and the residual cellularity of the tumor bed was estimated. The revised tumor size was calculated by multiplying the largest dimension of gross tumor-bed/fibrotic area with the tumor cellularity (compared with pretherapy biopsy) of the resection specimen. The percentage tumor size/volume reduction was calculated by subtracting revised tumor size from pretherapy size, divided by pretherapy size times 100 (see Table 1). Although this method may also be used for determining response within the lymph nodes, it was not used in this study as it requires pretherapy size and core biopsy of the lymph node, which was not available on all cases. Only presence or absence of tumor within lymph nodes was noted at post-therapy resection specimen to judge if pathologic response was complete or incomplete. Although immeasurable, if residual tumor was identified only in vascular 
Table 1 Worksheet for estimating tumor volume reduction in the breast secondary to neoadjuvant chemotherapy

Pretherapy tumor size

A1 Maximum dimension estimate in the following preferential order: MRI; Ultrasound; Mammogram; physical exam: $\mathrm{cm}$

Post-therapy tumor size

B1 Maximum dimension of tumor-bed/fibrotic area by gross exam: $\mathrm{cm}$

B2 Percentage cellularity (compared with pretherapy biopsy) of the tumor-bed/fibrotic area by microscopic exam:

B3 Revised tumor size after correcting for cellularity $(\mathrm{B} 1 \times \mathrm{B} 2)$ : $\mathrm{cm}$

Estimated primary tumor volume reduction

Pretherapy size (A1)-revised tumor size (B3)

Pretherapy size (A1)

spaces, it was considered as incomplete pathologic response. However, this is an extremely rare phenomenon and if thorough examination of the breast is performed, generally an intraparenchymal component is also identified. In few cases of inflammatory carcinoma where pretherapy size was not available, the size of tumor-bed/fibrotic area on gross exam was used to estimate the pretherapy size. All data were extracted from the pathology reports and no slides were reviewed for this study. Although no slides were reviewed for this study, all core biopsies and post-therapy resection specimens were analyzed by 13 breast pathologists with a median experience of $>5$ years in the field. The pretherapy tumor nuclear grade was also available from core biopsy reports. Pathologic complete response with respect to tumor nuclear grade was also analyzed.

Statistical analysis was performed using SPSS software version 16.0. For comparison of means, independent sample $t$-tests were performed. Univariate analysis was performed using $\chi^{2}$ and Fisher's exact tests to compare the differences in percentages between groups. A $P$-value of $<0.05$ was considered as significant.

\section{Results}

Of the total 104 cases, $48(46 \%)$ were classified as ERBB2, $30(29 \%)$ as LBHH and $26(25 \%)$ as LAHH. The patients in ERBB2 group were slightly older (median age 54) than in the LAHH (median age 48) and LBHH group (median age 48). The mean (median) pretherapy tumor size for ERBB2, LBHH and LAHH tumors were $4.1 \mathrm{~cm}(3.2 \mathrm{~cm}), 3.8 \mathrm{~cm}$ $(3.5 \mathrm{~cm})$ and $3.9(2.8 \mathrm{~cm})$, respectively. Tumors with nuclear grade 3 constituted 71\% (34 of 48) of ERBB2 tumors, $53 \%$ (16 of 30 ) of LBHH tumors and 58\% (15 of 26) of LAHH tumors. In all, 20 cases (19\%) showed immunohistochemical $2+$ expression for HER2 with unequivocal gene amplification. These cases were identified within all three categories: 5 belonged to ERBB2, 11 belonged to LBHH and 4 belonged to LAHH group. Of these 20 tumors, 3 showed pathologic complete response (2 within ERBB2 group and 1 within LBHH group).
Table 2 Pathologic complete response in various categories of HER2-positive tumors

\begin{tabular}{llcc}
\hline Tumor type & Criteria used & $p C R$ & No pCR \\
\hline ERBB2 & ER-/PR-/HER2+ & $25 / 48(52 \%)$ & $23 / 48(48 \%)$ \\
LBHH & ER+(W/M)/HER2+ & $10 / 30(33 \%)$ & $20 / 30(67 \%)$ \\
LAHH & ER+(S)/HER2+ & $2 / 26(8 \%)$ & $24 / 26(92 \%)$ \\
\hline
\end{tabular}

pCR: pathologic complete response; LBHH: Luminal B-HER2 Hybrid; LAHH: Luminal A-HER2 Hybrid; (S): strong; (W/M): weak to moderate.

Pathologic complete response in different types of HER2 + tumors is shown in Table 2. The difference in pathologic complete response rates among three categories was statistically significant ( $P$-value: 0.001). Univariate analysis for other available variables in predicting pathologic complete response showed that age, pretherapy tumor size and nuclear grade were not significant $(P$-values of $0.718,0.307$ and 0.549, respectively). Ki-67 labeling index was available in only 21 cases; however, it was not significant ( $P$-value of 0.307$)$ in predicting pathologic complete response. In contrast, the differences in ER and PR H-scores were predictive of pathologic complete response ( $P$-values of 0.001 and 0.002 , respectively). The tumors that achieved pathologic complete response showed a mean ER and PR H-scores of 47 and 13, respectively, versus an ER and PR H-scores of 122 and 64, respectively, in tumors that failed to achieve pathologic complete response.

The average percentage tumor volume reduction was significantly higher in ERBB2 tumors ( $P$-value: 0.001). The percentage of tumors with $\geq 50 \%$ tumor volume reduction was highest in ERBB2 tumors, followed by LBHH and LAHH tumors (Table 3). The ER and PR H-scores were similarly significant in predicting percentage tumor volume reduction ( $P$-values of 0.001 and 0.003 , respectively). Univariate analysis for other variables with respect to percentage volume reduction showed that age, pretherapy tumor size and nuclear grade were not significant ( $P$-values of $0.789,0.342$ and 0.230 , respectively).

As only hormone receptor semiquantitative scores were significant on univariate analysis and they 
Table 3 Pretherapy tumor size (burden) and percentage tumor volume reduction in various categories of HER2-positive tumors

\begin{tabular}{lccc}
\hline Tumor type & $\begin{array}{c}\text { Average } \\
\text { (median) } \\
\text { pretherapy } \\
\text { umor size }\end{array}$ & $\begin{array}{c}\text { Average } \\
\text { (median) } \\
\text { percentage } \\
\text { TVR }\end{array}$ & $\begin{array}{c}\text { Number } \\
\text { (percentage) } \\
\text { of cases with } \\
\geq 50 \% \text { TVR }\end{array}$ \\
\hline ERBB2 & $4.2 \mathrm{~cm}(3.2 \mathrm{~cm})$ & $86(100)$ & $43 / 48(90 \%)$ \\
LBHH & $3.8 \mathrm{~cm}(3.5 \mathrm{~cm})$ & $74(90)$ & $24 / 30(80 \%)$ \\
LAHH & $4.0 \mathrm{~cm}(2.8 \mathrm{~cm})$ & $64(70)$ & $15 / 26(58 \%)$
\end{tabular}

TVR: tumor volume reduction; LBHH: Luminal B-HER2 Hybrid; LAHH: Luminal A-HER2 Hybrid.

were the basis of tumor categorization, multivariate analysis was not feasible.

We also analyzed the data for any differences between tumors from the period before and after standardization of assessment of tumor volume reduction. There were 81 cases $(78 \%)$ that were examined in a non-standard fashion and 23 cases $(22 \%)$ subjected to standard protocol as described in the Materials and methods section. These two groups of tumors failed to show any statistical difference with respect to any variable, suggesting thoroughness of pathologic examination before institution of standardized protocol.

\section{Discussion}

Neoadjuvant or preoperative chemotherapy in operable breast cancer is increasingly used despite some disadvantages. The specific disadvantages are loss of prognostic information in node-negative tumors treated with preoperative chemotherapy, lack of reliability of negative margins in tumors that show assymetric reductions, potential for overtreating patients with clinically sizable tumors that are composed predominantly of noninvasive disease, the patient anxiety associated with leaving a resistant tumor in the patient while they are on neoadjuvant chemotherapy and loss of untreated primary tumors for other research purposes. Although the above arguments seem significant, there are several equally significant arguments in favor of neoadjuvant chemotherapy. Neoadjuvant chemotherapy provides an opportunity to examine the in vivo tumor responsiveness to particular chemotherapy regimen. Recent data in breast cancer also show that patient survival is not affected by timing of chemotherapy. Moreover, at least some degree of tumor volume reduction is achieved in a majority of breast cancer cases. Therefore, many patients for whom a mastectomy is initially proposed can be later changed to breast-conserving surgery after neoadjuvant chemotherapy. Last, but not the least, chemotherapy could be started for patients who are waiting for surgery because of pending gene testing results or other reasons.
Because of these reasons, neoadjuvant chemotherapy is increasingly used in breast cancer. However, the therapeutic regimens used are quite variable but most patients receive adriamycin- and/or taxanebased chemotherapy. In the last 5 years, trastuzumab is increasingly used in the neoadjuvant regimens for HER2 + tumors. Although the effectiveness of trastuzumab in HER2 + tumors is well established in the adjuvant setting, there is still paucity of studies analyzing response in the neoadjuvant setting.

The molecular studies using gene expression analysis have classified breast cancers into at least four distinct categories: Luminal A, Luminal B, ERBB2 (or HER2 enriched) and basal like. ${ }^{22-24}$ Further gene expression studies showed that these tumor classes respond differently to neoadjuvant chemotherapy. ${ }^{12}$ Immunohistochemical studies using surrogate markers for molecular classes showed similar results. Our previous study of 359 cases treated with neoadjuvant chemotherapy with only a handful of HER2 + patients receiving trastuzumab suggested that the extent of ER reactivity by immunohistochemistry could influence tumor response to trastuzumab. ${ }^{9}$ In order to test our preliminary findings, we expanded our analysis in this study to $>100$ HER2 + tumors treated with trastuzumab and also divided the tumors into three categories based on the extent of ER expression. Based on data from metastatic breast cancer studies, most HER2 + tumors are treated in a standard fashion at our institution with TCH chemotherapy since 2006 in the neoadjuvant setting. ${ }^{25}$ Therefore, there was negligible variability in chemotherapy regimen in the cases used in this study.

Our study clearly demonstrates that addition of trastuzumab to neoadjuvant chemotherapy regimen increases the rate of pathologic complete response by $\sim 10-20 \%$ in all varieties of HER2 + tumors. In our previous study, where most HER2 + tumors were treated with chemotherapy regimen that excluded trastuzumab, the pathologic complete response rates in ERBB2, LBHH and LAHH tumors were 33,8 and $0 \%$, respectively. In contrast, in this study, where all tumors were treated with trastuzumab-containing chemotherapy, the pathologic complete response rates in ERBB2, LBHH and LAHH tumors were 52,33 and $11 \%$, respectively. In comparison with one somewhat similar study of HER2 + tumors treated without trastuzumab, ${ }^{13}$ the pathologic complete response rate in ERBB2 tumors in this study appears to be slightly lower. However, it should be noted that most cases in our study underwent a very thorough gross and microscopic examination, and presence of even a single viable invasive tumor cell in the breast or the lymph node was considered as incomplete response. Although the pathologic complete response in ERBB2 tumors was $52 \%$, the mean and median percentage primary tumor volume reduction in ERBB2 tumor group was 86 and $100 \%$, respectively. 
In addition to pathologic complete response, we also analyzed tumor volume reduction in all tumors, where we utilized a very standard and practical approach in estimating tumor volume reduction. We believe the method reported in this study is at least as objective, if not more, compared with other methods ${ }^{26-28}$ and yet quite simple compared with MD Anderson method ${ }^{29}$ of estimating tumor volume reduction. This method made it possible to compare the differences between different tumor categories with confidence. Similar to pathologic complete response, maximum percentage tumor volume reduction was identified in ERBB2 tumors, followed by $\mathrm{LBHH}$ tumors and least in LAHH tumors. In addition to estimating absolute percentage tumor volume reduction for each case, we also analyzed the percentage of tumors in each group showing $\geq 50 \%$ tumor volume reduction. This information is very important from a surgeon's viewpoint in order to counsel patients about the likelihood of a breastconserving surgery after chemotherapy. Our results suggest that $\geq 50 \%$ tumor volume reduction can be expected in a majority of the HER2 + cases, with highest in ERBB2 and lowest in LAHH tumors. These findings clearly demonstrate that response to trastuzumab-containing chemotherapy in HER2 + tumors is inversely related to amount of ER expression.

It is well known that hormonal therapy in HER2 + tumors is not very effective, but the relative resistance to trastuzumab therapy in ER + /HER2 + tumors is under-recognized. Several studies have suggested that ER and HER2 cross-talk is responsible for this resistance when these tumors are treated with hormonal therapy alone. ${ }^{30,31}$ Recently, it has been shown that a combination of trastuzumab and hormonal therapy is superior than hormonal therapy alone. ${ }^{32-34}$ However, the question of relative resistance to trastuzumab therapy in ER +/HER2 + tumors still remains. Whether this is because of ER and HER2 cross-talk only or other growth factor receptors are also involved is a subject of active investigation. The involvement of other growth factors and their receptors may provide another target for these unique tumors. Some recent studies have shown significant synergy between ER and insulin growth factor (IGF) system that is demonstrable in both normal and malignant breast tissues. ${ }^{35-37}$ The activated ER complexes bind to estrogen-responsive elements in the promoter regions of the target genes that include IGF-1R. In the presence of unaltered ER pathway, ER ligands can promote cell growth via IGF-1R. In a similar manner, growth factors (EGF and IGF) have been shown to activate ER by phosphorylating the receptor. It is known that IGF-1R primarily activates the MAPK and PI3/Akt pathways and both of these signal transduction pathways phosphorylate the serine residue in the AF1 domain of the ER. ${ }^{38,39}$ This synergy obviously is possible if both ER and IGF-1R are expressed by tumor cells. Interestingly, we have recently shown that IGF receptor 1 (IGF-1R) is expressed at a significantly higher level in all ER + tumors (including ER + /HER2 +) compared with ER-/HER2 + tumors (Appl Immunohistochem Mol Morphol; in press). ${ }^{40}$ Similar findings were reported by Harris et al. ${ }^{41}$ If these findings are also supported by additional studies, IGF-1R may be another molecule that could be targeted with available antibodies ${ }^{42-45}$ in these difficult-to-treat ER + /HER2 + tumors, despite the presence of two well-known targets.

In summary, we have clearly shown that addition of trastuzumab to neoadjuvant chemotherapy regimen significantly increases the pathologic complete response rates in all HER2 + tumors. However, the benefit of trastuzumab is highest in ER-negative tumors and progressively decreases with increase in tumor ER expression. This information can be utilized to counsel patients considered for neoadjuvant chemotherapy and the same principle could be applied in the adjuvant setting. Generally, patients who achieve pathologic complete response have excellent prognosis compared with the ones that fail to achieve pathologic complete response. Whether addition of trastuzumab in neoadjuvant chemotherapy regimen would have the same effect will be analyzed in the years to come.

\section{Acknowledgement}

We thank Louise Mazur for clerical assistance.

\section{Disclosure/conflict of interest}

The authors declare no conflict of interest.

\section{References}

1 Fisher B, Bryant J, Wolmark N, et al. Effect of preoperative chemotherapy on the outcome of women with operable breast cancer. J Clin Oncol 1998;16: 2672-2685.

2 Mamounas EP, Fisher B. Preoperative (neoadjuvant) chemotherapy in patients with breast cancer. Semin Oncol 2001;28:389-399.

3 Mauri D, Pavlidis N, Ioannidis JP. Neoadjuvant versus adjuvant systemic treatment in breast cancer: a metaanalysis. J Natl Cancer Inst 2005;97:188-194.

4 Rastogi P, Anderson SJ, Bear HD, et al. Preoperative chemotherapy: updates of National Surgical Adjuvant Breast and Bowel Project Protocols B-18 and B-27. J Clin Oncol 2008;26:778-785.

5 Liu SV, Melstrom L, Yao K, et al. Neoadjuvant therapy for breast cancer. J Surg Oncol 2010;101:283-291.

6 Fisher B, Brown A, Mamounas E, et al. Effect of preoperative chemotherapy on local-regional disease in women with operable breast cancer: findings from National Surgical Adjuvant Breast and Bowel Project B-18. J Clin Oncol 1997;15:2483-2493. 
7 Mamounas EP. Neoadjuvant chemotherapy for operable breast cancer: is this the future? Clin Breast Cancer 2003;4(Suppl 1):S10-S19.

8 Wolmark N, Wang J, Mamounas E, et al. Preoperative chemotherapy in patients with operable breast cancer: nine-year results from National Surgical Adjuvant Breast and Bowel Project B-18. J Natl Cancer Inst Monogr 2001;30:96-102.

9 Bhargava R, Beriwal S, Dabbs DJ, et al. Immunohistochemical surrogate markers of breast cancer molecular classes predicts response to neoadjuvant chemotherapy: a single institutional experience with 359 cases. Cancer 2010;116:1431-1439.

10 Carey LA, Dees EC, Sawyer L, et al. The triple negative paradox: primary tumor chemosensitivity of breast cancer subtypes. Clin Cancer Res 2007;13:2329-2334.

11 Rody A, Karn T, Solbach C, et al. The erbB2+ cluster of the intrinsic gene set predicts tumor response of breast cancer patients receiving neoadjuvant chemotherapy with docetaxel, doxorubicin and cyclophosphamide within the GEPARTRIO trial. Breast 2007; 16:235-240.

12 Rouzier R, Perou CM, Symmans WF, et al. Breast cancer molecular subtypes respond differently to preoperative chemotherapy. Clin Cancer Res 2005;11: 5678-5685.

13 Andre F, Mazouni C, Liedtke C, et al. HER2 expression and efficacy of preoperative paclitaxel/FAC chemotherapy in breast cancer. Breast Cancer Res Treat 2008;108:183-190.

14 Buzdar AU, Ibrahim NK, Francis D, et al. Significantly higher pathologic complete remission rate after neoadjuvant therapy with trastuzumab, paclitaxel, and epirubicin chemotherapy: results of a randomized trial in human epidermal growth factor receptor 2-positive operable breast cancer. J Clin Oncol 2005;23: 3676-3685.

15 Gianni L, Eiermann W, Semiglazov V, et al. Neoadjuvant chemotherapy with trastuzumab followed by adjuvant trastuzumab versus neoadjuvant chemotherapy alone, in patients with HER2-positive locally advanced breast cancer (the NOAH trial): a randomised controlled superiority trial with a parallel HER2-negative cohort. Lancet 2010;375:377-384.

16 Untch M, Rezai M, Loibl S, et al. Neoadjuvant treatment with trastuzumab in HER2-positive breast cancer: results from the GeparQuattro study. J Clin Oncol 2010;28:2024-2031.

17 Lemieux J, Clemons M, Provencher L, et al. The role of neoadjuvant her2-targeted therapies in her2-overexpressing breast cancers. Curr Oncol 2009;16:48-57.

18 Wildiers H, Neven P, Christiaens MR, et al. Neoadjuvant capecitabine and docetaxel (plus trastuzumab): an effective non-anthracycline-based chemotherapy regimen for patients with locally advanced breast cancer. Ann Oncol 2010. e-pub ahead of print: PMID 20709813.

19 Buzdar AU, Valero V, Ibrahim NK, et al. Neoadjuvant therapy with paclitaxel followed by 5 -fluorouracil, epirubicin, and cyclophosphamide chemotherapy and concurrent trastuzumab in human epidermal growth factor receptor 2-positive operable breast cancer: an update of the initial randomized study population and data of additional patients treated with the same regimen. Clin Cancer Res 2007;13:228-233.

20 Bhargava R, Striebel J, Beriwal S, et al. Prevalence, morphologic features and proliferation indices of breast carcinoma molecular classes using immunohistochemical surrogate markers. Int J Clin Exp Pathol 2009;2:444-455.

21 McCarty Jr KS, Miller LS, Cox EB, et al. Estrogen receptor analyses. Correlation of biochemical and immunohistochemical methods using monoclonal antireceptor antibodies. Arch Pathol Lab Med 1985; 109:716-721.

22 Perou CM, Sorlie T, Eisen MB, et al. Molecular portraits of human breast tumours. Nature 2000;406: 747-752.

23 Sorlie T, Perou CM, Tibshirani R, et al. Gene expression patterns of breast carcinomas distinguish tumor subclasses with clinical implications. Proc Natl Acad Sci USA 2001;98:10869-10874.

24 Sorlie T, Tibshirani R, Parker J, et al. Repeated observation of breast tumor subtypes in independent gene expression data sets. Proc Natl Acad Sci USA 2003;100:8418-8423.

25 Robert N, Leyland-Jones B, Asmar L, et al. Randomized phase III study of trastuzumab, paclitaxel, and carboplatin compared with trastuzumab and paclitaxel in women with HER-2-overexpressing metastatic breast cancer. J Clin Oncol 2006;24:2786-2792.

26 Kurosumi M. Significance and problems in evaluations of pathological responses to neoadjuvant therapy for breast cancer. Breast Cancer 2006;13:254-259.

27 Ogston KN, Miller ID, Payne S, et al. A new histological grading system to assess response of breast cancers to primary chemotherapy: prognostic significance and survival. Breast 2003;12:320-327.

28 Smith IC, Heys SD, Hutcheon AW, et al. Neoadjuvant chemotherapy in breast cancer: significantly enhanced response with docetaxel. J Clin Oncol 2002;20: 1456-1466.

29 Symmans WF, Peintinger F, Hatzis C, et al. Measurement of residual breast cancer burden to predict survival after neoadjuvant chemotherapy. J Clin Oncol 2007;25:4414-4422.

30 Osborne CK, Shou J, Massarweh S, et al. Crosstalk between estrogen receptor and growth factor receptor pathways as a cause for endocrine therapy resistance in breast cancer. Clin Cancer Res 2005;11: 865s-8870s.

31 Shou J, Massarweh S, Osborne CK, et al. Mechanisms of tamoxifen resistance: increased estrogen receptorHER2/neu cross-talk in ER/HER2-positive breast cancer. J Natl Cancer Inst 2004;96:926-935.

32 Cortes J, Baselga J. How to treat hormone receptorpositive, human epidermal growth factor receptor 2-amplified breast cancer. J Clin Oncol 2009;27: 5492-5494.

33 Johnston S, Pippen Jr J, Pivot X, et al. Lapatinib combined with letrozole versus letrozole and placebo as first-line therapy for postmenopausal hormone receptor-positive metastatic breast cancer. J Clin Oncol 2009;27:5538-5546.

34 Kaufman B, Mackey JR, Clemens MR, et al. Trastuzumab plus anastrozole versus anastrozole alone for the treatment of postmenopausal women with human epidermal growth factor receptor 2-positive, hormone receptor-positive metastatic breast cancer: results from the randomized phase III TAnDEM study. J Clin Oncol 2009;27:5529-5537.

35 Fagan DH, Yee D. Crosstalk between IGF1R and estrogen receptor signaling in breast cancer. J Mammary Gland Biol Neoplasia 2008;13:423-429. 
36 Ruan W, Catanese V, Wieczorek R, et al. Estradiol enhances the stimulatory effect of insulin-like growth factor-I (IGF-I) on mammary development and growth hormone-induced IGF-I messenger ribonucleic acid. Endocrinology 1995;136:1296-1302.

37 Stewart AJ, Johnson MD, May FE, et al. Role of insulinlike growth factors and the type I insulin-like growth factor receptor in the estrogen-stimulated proliferation of human breast cancer cells. J Biol Chem 1990;265: 21172-21178.

38 Kato S, Endoh H, Masuhiro Y, et al. Activation of the estrogen receptor through phosphorylation by mitogenactivated protein kinase. Science 1995;270:1491-1494.

39 Simoncini T, Hafezi-Moghadam A, Brazil DP, et al. Interaction of oestrogen receptor with the regulatory subunit of phosphatidylinositol-3-OH kinase. Nature 2000;407:538-541.

40 Bhargava R, Beriwal S, Dabbs DJ. IGF-1R expression in normal breast tissue, benign, proliferative, in-situ breast lesions, and invasive breast carcinoma. Cancer Res 2009;69(24 Suppl): 662S, Abstract no. 3051.

41 Harris LN, You F, Schnitt SJ, et al. Predictors of resistance to preoperative trastuzumab and vinorelbine for HER2-positive early breast cancer. Clin Cancer Res 2007;13:1198-1207.

42 Burtrum D, Zhu Z, Lu D, et al. A fully human monoclonal antibody to the insulin-like growth factor I receptor blocks ligand-dependent signaling and inhibits human tumor growth in vivo. Cancer Res 2003;63:8912-8921.

43 Ji QS, Mulvihill MJ, Rosenfeld-Franklin M, et al. A novel, potent, and selective insulin-like growth factor-I receptor kinase inhibitor blocks insulin-like growth factor-I receptor signaling in vitro and inhibits insulin-like growth factor-I receptor dependent tumor growth in vivo. Mol Cancer Ther 2007;6: 2158-2167.

44 Maloney EK, McLaughlin JL, Dagdigian NE, et al. An anti-insulin-like growth factor I receptor antibody that is a potent inhibitor of cancer cell proliferation. Cancer Res 2003;63:5073-5083.

45 Wang Y, Hailey J, Williams D, et al. Inhibition of insulin-like growth factor-I receptor (IGF-IR) signaling and tumor cell growth by a fully human neutralizing anti-IGF-IR antibody. Mol Cancer Ther 2005;4: 1214-1221. 